\title{
BOUNDED SETS IN (LF)-SPACES
}

\author{
JOSÉ BONET AND CARMEN FERNÁNDEZ
}

(Communicated by Theodore Gamelin)

\begin{abstract}
The behaviour of bounded sets is important in the theory of countable inductive limits of Fréchet spaces, the (LF)-spaces, and its applications. An (LF)-space is called regular if every bounded set is contained and bounded in one of the steps. In the present paper necessary conditions and sufficient conditions are given for the regularity of an (LF)-space. The conditions are expressed in terms of the behaviour of the neighbourhoods of the steps. It is proved that the conditions are equivalent for (LF)-spaces of sequences or of continuous functions.
\end{abstract}

The purpose of the present article is to give necessary (and also sufficient) conditions to ensure that an (LF)-space satisfies that every bounded set is contained and bounded in some of the steps. The countable inductive limits of Fréchet spaces, called (LF)-spaces, were thoroughly studied by Dieudonné and Schwartz [7] and by Grothendieck [8]. They were motivated by their relevance in the theory of distributions of Schwartz and its applications to partial differential equations. More recently several authors have analyzed the structure of (LF)-spaces and of (LF)-spaces of sequences and of continuous or holomorphic functions. These spaces are important in connection with applications to spaces of ultradistributions and convolution equations. We refer to [1], [9] and [17] for excellent presentations of the theory of (LF)-spaces.

The space $(E, t)=\operatorname{ind}_{n}\left(E_{n}, t_{n}\right)$ is an (LF)-space if $\left(E_{n}, t_{n}\right)_{n \in \mathbb{N}}$ is an increasing sequence of Fréchet spaces with continuous inclusions $\left(E_{n}, t_{n}\right) \subset$ $\left(E_{n+1}, t_{n+1}\right), E=\bigcup_{n \in \mathbb{N}} E_{n}$ and $(E, t)$ is endowed with the finest locally convex topology such that the injections from $\left(E_{n}, t_{n}\right)$ into $E$ are continuous. Here an (LF)-space is always assumed to be Hausdorff. If every step $\left(E_{n}, t_{n}\right)$ is a Banach space, then the inductive limit $E$ is called an (LB)-space. In what follows, for each $n \in \mathbb{N}$, we let $\left(U_{n, k}\right)_{k \in \mathbb{N}}$ denote a basis of absolutely convex 0 -neighbourhoods in $E_{n}$. We will assume without loss of generality

(a) $U_{n, k} \supset U_{n, k+1} \forall n, k \in \mathbb{N}$; (b) $U_{n, k} \subset U_{n+1, k} \forall n, k \in \mathbb{N}$.

We will use $n, m, \mu, \nu, M, N$ for natural numbers in the first index (steps) and $k, l, K, L$ for natural numbers in the second index (neighbourhoods in a

Received by the editors November 18, 1993 and, in revised form, May 18, 1994.

1991 Mathematics Subject Classification. Primary 46A13, 46M40.

Key words and phrases. Fréchet spaces, (LF)-spaces, regularity and completeness, condition (M) of Retakh, Köthe sequence (LF)-spaces, weighted inductive limits.

The research of this article was partially supported by DGICYT Proyecto no. PB91-0538. 
fixed step). If $E_{n}$ is a Banach space with unit ball $B_{n}$, we assume $2 B_{n} \subset B_{n+1}$ for each $n \in \mathbb{N}$ and we take $U_{n, k}:=2^{-k+1} B_{n}$ for each $k \in \mathbb{N}$. Our notation for locally convex spaces is standard. See e.g. [14].

The behaviour of bounded sets is very important in (LF)-spaces. An (LF)space $E$ is called regular if every bounded subset $B$ of $E$ is contained and bounded in some step $E_{n}$. Grothendieck proved that an (LF)-space is regular if and only if it is locally complete (see e.g. [14, Chapter 5]). Accordingly, every complete (LF)-space is regular and it is an open problem, due to Grothendieck, whether every regular (LF)-space is complete. See e.g. [1, p. 78]. The structure of (LB)-spaces is quite well understood (see [1, 3, 4]). Several authors have investigated regularity properties of (LF)-spaces (Bosch, Kucera, McKennon and Qiu Jing Hui $[10,11,12,15,16]$ have treated these questions). The important work of Vogt [17] presented Palamodov's theory of acyclic (and weakly acyclic) spaces, avoiding the homological tools, and versions of Retakh's conditions (and their relation to the regularity) which are suitable for applications and for evaluation in concrete cases. Vogt [17] also introduced and studied the Köthe (LF)-spaces of sequences $E^{p} \quad(1 \leq p \leq \infty)$. He characterized their regularity and completeness. These spaces appear in the study of duals of kernels of convolution equations on spaces of ultradistributions. Bierstedt and the first author [2] studied weighted (LF)-spaces of continuous functions. The characterization of regular (LF)-spaces of Moscatelli type was given in [6, 13]. The (LB)-spaces of Moscatelli type were studied in [5].

Our aim in this pape is to prove necessary (and also sufficient) conditions for an (LF)-space to be regular. They must be considered as extensions of the following well-known characterization of regular (LB)-spaces: an (LB)-space $E=\operatorname{ind}_{n} E_{n}$ is regular if and only if for all $n \in \mathbb{N}$ there is $m \geq n$ such that $\overline{B_{n}} \subset B_{m}$ (the closure taken in $E$ ). We will also recover the necessary condition (wQ) of Vogt [17]. Our proof is different from Vogt's. Moreover the consideration of closures permits also to give sufficient conditions, which are equivalent to the necessary ones for concrete (LF)-spaces of sequences and continuous functions. Our main result is the following theorem which improves all the known results.

Theorem 1. Let $E=\operatorname{ind}_{n} E_{n}$ be an ( $\left.L F\right)$-space. Consider the following conditions:

(1) There is an increasing sequence $\left(U_{n}\right)_{n \in \mathbb{N}}$ of absolutely convex closed 0neighbourhoods $U_{n}$ in $E_{n}$ such that

(1.a) $\forall n \exists m \geq n: \overline{U_{n}} \subset U_{m}$, the closure taken in $E$,

(1.b) $\forall n \exists m \geq n:$ if $A \subset U_{n}$ is bounded in $E$, then $A$ is bounded in $E_{m}$.

(2) $E$ is regular.

(3) The following two conditions are satisfied by the neighbourhoods $\left(U_{n, k}\right)_{n, k \in \mathbb{N}}$ :

(3.a) $\forall n \exists m \geq n \forall l \exists L: \overline{U_{n, L}} \subset U_{m, l}$, the closure taken in $E$,

(3.b) $\forall n \exists m \geq n, k:$ if $A \subset U_{n, k}$ is bounded in $E$, then $A$ is bounded in $E_{m}$.

Then (1) implies (2) and (2) implies (3).

Proof. All the closures in this proof are taken in $E$.

(1) $\Rightarrow(2)$. Let $A$ be a bounded subset of $E$. By a result of Makarov (see 
e.g. $[14,8.5 .20])$, there is $\nu \in \mathbb{N}$ with $A \subset \nu \overline{U_{\nu}}$. By (1.a) we find $\mu \geq \nu$ with $\bar{U}_{\nu} \subset U_{\mu}$. We apply (1.b) for $n=\mu$ to find $m$. We have $\nu^{-1} A \subset U_{\mu}$ and $\nu^{-1} A$ is bounded in $E$. By (1.b) $A$ is bounded in $E_{m}$.

$(2) \Rightarrow(3)$. We assume without loss of generality that $2 U_{n, k+1} \subset U_{n, k}$ for all $n, k$. We prove (3.a) by contradiction. If (3.a) is not satisfied we have

$\exists n \forall m \geq n \exists l(m): \overline{U_{n, m+L}}$ is not contained in $L U_{m, l(m)}$ for all $L \in \mathbb{N}$.

For each $L \in \mathbb{N}$ we select $x_{m, L} \in \overline{U_{n, m+L}}$ with $x_{m, L} \notin L U_{m, l(m)}$ and we define $B:=\left\{x_{m, L} ; m, L \in \mathbb{N}\right\}$. We show that $B$ is bounded in $E$. In fact, if $U$ is a closed absolutely convex 0 -neighbourhood in $E$, there is $k \in \mathbb{N}$ with $\overline{U_{n, k}} \subset U$. This implies $x_{m, L} \in U$ for all $m \geq k$ and all $L \in \mathbb{N}$. On the other hand, if $1 \leq m<k, x_{m, L} \in \overline{U_{n, k}}$ except for a finite number of $L$ 's. This implies that $B$ is bounded in $E$. Since $E$ is regular, $B$ must be bounded in some $E_{m}$, hence $B$ is absorbed by $U_{m, l(m)}$, a contradiction.

We also prove (3.b) by contradiction. If it does not hold, there is $n \in \mathbb{N}$ such that

$$
\forall m \geq n \forall k \exists A \subset U_{n, k} \text { bounded in } E \text { but not bounded in } E_{m} .
$$

Without loss of generality we assume $n=1$. Then for all $k \in \mathbb{N}$ there is a bounded subset $A_{k}$ of $E$ with $A_{k} \subset U_{1, k}$ and there is $l(k) \in \mathbb{N}$ such that $A_{k}$ is not absorbed by $U_{k+1, l(k)}$. We select $\left(x_{k, j}\right)_{j \in \mathbb{N}} \subset A_{k}$ with $x_{k, j} \notin j U_{k+1, l(k)}$ for each $j \in \mathbb{N}$. The set $A:=\left\{x_{k, j}: k, j \in \mathbb{N}\right\}$ is bounded in $E$. Indeed, if $U$ is a closed absolutely convex 0 -neighbourhood in $E$, there is $k(0) \in \mathbb{N}$ with $U_{1, k(0)} \subset U$, hence $x_{k, j} \in U$ if $k \geq k(0)$. Moreover $\left\{x_{k, j} ; 1 \leq k<k(0), j \in\right.$ $\mathbb{N}\} \subset A_{1} \cup \cdots \cup A_{k(0)-1}$ is bounded in $E$. By construction $A$ is not bounded in each $E_{m}$. This contradicts the regularity of $E$.

The implication "(1) implies (2)" in the theorem above improves [16, Theorems 4 and 5]. Our next corollary follows from the implication "(2) implies (3.a)" in Theorem 1 .

Corollary 2. If $E=$ ind $_{n} E_{n}$ is a regular $(L F)$-space, then for every $n \in \mathbb{N}$ there is $m \geq n$ such that if $B$ is a bounded subset of $E_{n}$, its closure in $E$ is bounded in $E_{m}$.

An (LF)-space $(E, t)=\operatorname{ind}_{n}\left(E_{n}, t_{n}\right)$ is said to satisfy condition (M) of $\operatorname{Re}$ takh if there is an increasing sequence $\left(U_{n}\right)_{n \in \mathbb{N}}$ of 0 -neighbourhoods $U_{n}$ in $\left(E_{n}, t_{n}\right)$ such that

$\forall n \exists m \geq n \forall \mu \geq m: t_{\mu}$ and $t_{m}$ induce the same topology on $U_{n}$.

We can assume without loss of generality that $U_{n}$ is closed in $\left(E_{n}, t_{n}\right)$ for each $n \in \mathbb{N}$. This condition is thoroughly discussed in [17], where (LF)-spaces which satisfy condition (M) are called acyclic. Acyclic (LF)-spaces are important in connection with limit and well-located subspaces of (LF)-spaces and their applications to convolution equations. See [17]. Vogt [17] introduced the following condition (wQ), necessary for regularity, which is easy to evaluate in concrete examples. The (LF)-space $E=\operatorname{ind}_{n} E_{n}$ is said to satisfy condition (wQ) if

$$
\forall n \exists m \geq n, k \forall l, \mu \geq m \exists L, S: U_{n, k} \cap U_{\mu, L} \subset S U_{m, l} \text {. }
$$

Every (LB)-space satisfies condition (wQ). 
Observation. (1) The following conditions are equivalent for an (LF)-space $E=$ ind $_{n} E_{n}$ : (i) $E$ satisfies condition (wQ); (ii) for every $n$ there are $m \geq n$ and $k$ such that for each $\mu \geq m$, every bounded subset of $E_{\mu}$ which is contained in $U_{n, k}$ is also bounded in $E_{m}$. Moreover condition (3.b) in Theorem 1 implies (ii). Accordingly we obtain a different proof of [17, 4.7]: every regular (LF)space satisfies condition (wQ).

We prove the equivalence of (i) and (ii). We only have to show that (ii) implies (i). Given $n$, we choose $m$ and $k$ as in (ii) and suppose there are $l$ and $\mu$ such that $U_{n, k} \cap U_{\mu, L}$ is not contained in $S U_{m, l}$ for all $L, S$. For each $L \in \mathbb{N}$ we select $x_{L} \in\left(U_{n, k} \cap U_{\mu, L}\right) \backslash L U_{m, l}$. Then $\left\{x_{L} ; L \in \mathbb{N}\right\}$ is bounded in $E_{\mu}$, it is contained in $U_{n, k}$, but it is not bounded in $E_{m}$. A contradiction.

(2) If the (LF)-space $E=\operatorname{ind}_{n} E_{n}$ satisfies condition (M), then it also satisfies condition (1) of Theorem 1. Indeed, let $\left(U_{n}\right)_{n \in \mathbb{N}}$ be the increasing sequence of $E_{n}$-closed neighbourhoods $U_{n}$ in $E_{n}$ given by condition (M). By [17, 2.11], we may assume that if $m \geq n$ is selected as in (M), then $E$ and $E_{m}$ induce the same topologies on $U_{n}$. To check (1.a), given $n$, we select $m$ as in (M). If $x$ belongs to $\overline{U_{n}}$ (closure taken in $E$ ), there is a net $\left(x_{i}\right) \subset U_{n}$ converging to $x$ in $E$. Thus $\left(x_{i}\right)$ is a Cauchy net in $U_{n}$ for the $E_{m}$-topology. This implies that $x_{i}$ converges to $x$ in $E_{m}$, hence $x \in U_{m}$. Now we prove (1.b) and, given $n$, we select again $m \geq n$ as in condition (M). Assume $A \subset U_{n}$ is bounded in $E$ but not bounded in $E_{m}$. We can find a sequence $\left(x_{k}\right)$ in $A$ and a sequence $\left(\lambda_{k}\right)$ tending to 0 in the scalar field such that $\lambda_{k} x_{k}$ does not tend to 0 in $E_{m}$. This contradicts $(\mathrm{M})$, since $\lambda_{k} x_{k}$ goes to 0 in $E$.

It is easy to see that the three conditions in Theorem 1 are equivalent for an (LB)-space $E$. In fact they are equivalent to the following condition: for all $n$ there is $m \geq n$ such that the closure of $B_{n}$ in $E$ is contained in $B_{m}$. In the rest of the article we will show that they are also equivalent for Köthe (LF)-spaces of sequences, for weighted (LF)-spaces of continuous functions and for (LF)-spaces of Moscatelli type.

We will let $X$ denote a locally compact Hausdorff topological space. For every $n \in \mathbb{N}, V_{n}=\left(v_{n, k}\right)_{k \in \mathbb{N}}$ is a sequence of strictly positive continuous functions on $X$. We denote by $\mathscr{V}$ the sequence $\left(V_{n}\right)_{n \in \mathbb{N}}$, and we assume that the following two conditions are satisfied:

(1) $v_{n, k}(x) \leq v_{n, k+1}(x) \quad \forall n, k \in \mathbb{N} \quad \forall x \in X$,

(2) $v_{n, k}(x) \geq v_{n+1, k}(x) \quad \forall n, k \in \mathbb{N} \quad \forall x \in X$.

For each $n \in \mathbb{N}$, we define the following weighted Fréchet spaces of continuous functions:

$$
\begin{array}{r}
C V_{n}(X):=\left\{f \in C(X) ; \forall k \in \mathbb{N}: \sup _{x \in X} v_{n, k}(x)|f(x)|<\infty\right\}, \\
C\left(V_{n}\right)_{0}(X):=\{f \in C(X) ; \forall k \in \mathbb{N} \forall \varepsilon>0 \exists K \subset X \text { compact: } \\
\left.v_{n, k}(x)|f(x)| \leq \varepsilon \forall x \in X \backslash K\right\} .
\end{array}
$$

We assume without loss of generality that $\left(U_{n, k}\right)_{k \in \mathbb{N}}$,

$$
U_{n, k}:=\left\{f \in C V_{n}(X) ; \sup _{x \in X} v_{n, k}(x)|f(x)| \leq 1\right\} \quad \text { for } k \in \mathbb{N},
$$

forms a basis of 0 -neighbourhoods in the space $C V_{n}(X)$ for each $n \in \mathbb{N}$. The weighted $(L F)$-spaces of continuous functions [2] are defined by

$$
\mathscr{V} C(X):=\operatorname{ind}_{n} C V_{n}(X) \text { and } \mathscr{V}_{0} C(X):=\operatorname{ind}_{n} C\left(V_{n}\right)_{0}(X)
$$


If $X=\mathbb{N}$ endowed with the discrete topology is considered as an index set and $1 \leq p<\infty$, we can define the Köthe (LF)-spaces of sequences [17] by $E^{p}(\mathscr{V}):=\operatorname{ind}_{n} E_{n}^{p}$ with

$$
E_{n}^{p}:=\left\{x=\left(x_{i}\right)_{i \in \mathbb{N}} ; \forall k \in \mathbb{N} \sum_{i \in \mathbb{N}}\left(v_{n, k}(i)\left|x_{i}\right|\right)^{p}<\infty\right\}
$$

endowed with the canonical topology.

The sequence $\mathscr{V}$ is said to satisfy condition (wQ) $)_{\mathscr{V}}$ (cf. [17]) if

$$
\begin{gathered}
\forall n \exists m \geq n, k \forall \mu \geq m, l \exists L, S \forall x \in X: \\
v_{m, l}(x) \leq S \max \left(v_{n, k}(x), v_{\mu, L}(x)\right) .
\end{gathered}
$$

Proposition 3. The three conditions in Theorem 1 are equivalent for Köthe $(L F)$-spaces of sequences $E^{p}(\mathscr{V})$ and for weighted $(L F)$-spaces of continuous functions $\mathscr{V} C(X)$ and $\mathscr{V}_{0} C(X)$.

Proof. First we consider the case of $\mathscr{V} C(X)$. Suppose that it satisfies condition (3) of Theorem 1. Condition (3.b) and $[2,1.2]$ imply that $\mathscr{V}$ satisfies condition $(w Q)_{\mathscr{V}}$ (see observation (1) above). By $[2,2.4]$ this implies that $\mathscr{V} C(X)$ is regular and, by the remarks after Definition 2.1 in [2], $\mathscr{V}$ satisfies the following stronger condition

$\left(w Q^{*}\right)$

$$
\begin{aligned}
\exists(k(\nu))_{\nu \in \mathbb{N}} \forall n \exists m \geq n \forall \mu \geq m, l \exists L, S: \\
v_{m, l} \leq S \max \left(\min _{1 \leq \nu \leq n} v_{\nu, k(\nu)}, v_{\mu, L}\right) .
\end{aligned}
$$

We write $w_{n}:=\min _{1 \leq \nu \leq n} v_{\nu, k(\nu)}$ and $U_{n}:=\left\{f \in C V_{n}(X) ; \sup _{x \in X} w_{n}(x)|f(x)|\right.$ $\leq 1\}$ for all $n \in \mathbb{N}$. Then the sequence $\left(U_{n}\right)_{n \in \mathbb{N}}$ satisfies (1.a) and (1.b) by $\left(w Q^{*}\right)$ and (the proof of $[2,2.6]$.

Now we assume that $\mathscr{V}_{0} C(X)$ satisfies condition (3) of Theorem 1. It is easy to see that (3.b) implies that $\mathscr{V}$ satisfies condition (wQ) $\mathscr{V}$. To conclude, by the observation (2) above and [2,3.3], it is enough to show that $\mathscr{V}$ satisfies the countable regularly decreasing condition (CRD) of $[2,3.2(5)]$. Now the proof of the implication "(12) implies (1)" in [2,3.3] permits the conclusion, since condition (3.a) implies the claim in part (b) of that proof.

Finally the proof in the case of Köthe (LF)-spaces of sequences $E^{p}(\mathscr{V})$ is very similar to the proof of the case of $\mathscr{V} C(X)$.

We recall the definition of an (LF)-space of Moscatelli type associated with $l_{\infty}, Y \subset X$ [6] (see [13] for the case of an arbitrary normal Banach sequence space $L$ instead of $l_{\infty}$.) We fix two Fréchet spaces $Y \subset X$ with continuous inclusion and we set $E_{n}:=l_{\infty}\left((X)_{k<n},(Y)_{k \geq n}\right)$, which is a Fréchet space for the canonical topology. We define $E:=\operatorname{ind}_{n} E_{n}$. In [6, Prop. 6] it is proved that $E$ is regular if and only if $Y$ admits an $X$-closed 0 -neighborhood. We have

Proposition 4. The three conditions of Theorem 1 are equivalent for $(L F)$-spaces of Moscatelli type.

Proof. Let $E$ be the (LF)-space of Moscatelli type associated with $l_{\infty}, Y \subset X$. The proof for arbitrary $L$ instead of $l_{\infty}$ is similar. We assume that $E$ has condition (3) in Theorem 1. We first show that there is a 0 -neighbourhood $V$ 
in $Y$ whose closure in $X$ is contained in $Y$. If this is not the case, we find a basis of 0 -neighbourhoods $\left(V_{n}\right)_{n \in \mathbb{N}}$ in $Y$ such that for every $m \in \mathbb{N}$ there is $x_{m} \in \overline{V_{m}} \backslash Y$, the closure taken in $X$. We set

$$
x^{m}:=\left(\delta_{m, j} x_{m}\right)_{j \in \mathbb{N}} \in \bigoplus_{\mathbb{N}} X \subset E \quad \text { for } m \in \mathbb{N}
$$

Then $x^{m} \notin E_{m}$ but $x^{m} \in \overline{V_{m}^{\mathbb{N}} \cap l_{\infty}(Y)}$ for each $m \in \mathbb{N}$, the closure taken in $E$. This contradicts (3.a) for $n=1$. In particular we have shown that $E$ is regular by [6, Prop. 6].

Next, to prove (1), we select an $X$-closed absolutely convex 0 -neighbourhood $V$ in $Y$ such that every $X$-bounded subset of $V$ is also $Y$-bounded (cf. [6, Lemma 5]) and we define

$$
\mathscr{U}_{n}:=\bigoplus_{k=1}^{n-1} X \times V^{\mathbb{N}} \cap l_{\infty}(Y) \text { for all } n \in \mathbb{N} .
$$

It is easy to see that $\mathscr{U}_{n}$ is closed in $E$ and that the sequence $\left(\mathscr{U}_{n}\right)_{n \in \mathbb{N}}$ is increasing. This implies (1.a). To check (1.b) we fix $n \in \mathbb{N}$ and a bounded subset $\mathscr{A}$ of $E$ such that $\mathscr{A} \subset \mathscr{U}_{n}$. Since $E$ is regular, $\mathscr{A}$ is bounded in some $E_{m}$ with $m \geq n$. From this it easily follows that $\mathscr{A}$ is in fact bounded in $E_{n}$.

\section{REFERENCES}

1. K. D. Bierstedt, An introduction to locally convex inductive limits, Functional Analysis and its Applications (Nice 1986), Singapore, New Jersey and Hong Kong, 1988, pp. 35-133.

2. K. D. Bierstedt and J. Bonet, Weighted (LF)-spaces of continuous functions, Math. Nachr. 165 (1994), 25-48.

3. K. D. Bierstedt, R. Meise, and W. H. Summers, A projective description of weighted inductive, limits, Trans. Amer. Math. Soc. 272 (1982), 107-160.

4. __ Köthe sets and Köthe sequence spaces, Functional Analysis, Holomorphy and Approximation Theory, North-Holland Math. Stud., vol. 71, North-Holland, Amsterdam and New York, 1982, pp. 27-91.

5. J. Bonet and S. Dierolf, On (LB)-spaces of Moscatelli type, Doğa Mat. 13 (1989), 9-33.

6. J. Bonet, S. Dierolf, and C. Fernández, On two classes of ( $L F)$-spaces, Portugal. Mat. 49 (1992), 109-130.

7. J. Dieudonné and L. Schwartz, La dualité dans les espaces $(F)$ et $(L F)$, Ann. Inst. Fourier (Grenoble) 1 (1954), 47-65.

8. A. Grothendieck, Produits tensoriels topologiques et espaces nucléaires, Mem. Amer. Math. Soc., no. 16, Amer. Math. Soc., Providence, RI, 1955.

9. K. Floret, Some aspects of the theory of locally convex inductive limits, Functional Analysis, Surveys and Recent Results II, North-Holland Math. Stud., vol. 38, North-Holland, Amsterdam and New York, 1980, pp. 205-237.

10. J. Kucera and K. McKennon, Bounded sets in inductive limits, Proc. Amer. Math. Soc. 69 (1978), 62-64.

11. _ Dieudonné-Schwartz theorem on bounded sets in inductive limits, Proc. Amer. Math. Soc. 78 (1980), 366-368.

12. J. Kucera and C. Bosch, Dieudonné-Schwartz theorem on bounded sets in inductive limits II, Proc. Amer. Mat. Soc. 86 (1982), 392-394.

13. Y. Melendez, On generalized (LF)-spaces of Moscatelli type, Doğa Mat. 15 (1991), 172-192. 
14. P. Pérez Carreras and J. Bonet, Barrelled locally convex spaces, North-Holland Math. Stud., vol. 131, North-Holland, Amsterdam and New York, 1987.

15. Qiu Jing Hui, Dieudonné Schwartz theorem in inductive limits of metrizable spaes, Proc. Amer. Math. Soc. 92 (1984), 255-257.

16. _ Dieudonné-Schwartz theorem in inductive limits of metrizable spaces II, Proc. Amer. Math. Soc. 108 (1990), 172-175.

17. D. Vogt, Regularity properties in $(L F)$-spaces, Progress in Functional Analysis, NorthHolland Math. Stud., vol. 170, North-Holland, Amsterdam and New York, 1992, pp. 57-84.

Departamento de Matemática Aplicada, E. T. S. Arquitectura, Universidad PolitécNiCA De Valencia, E-46071 Valencia, Spain

Current address, J. Bonet: FB17 Mathematik, Universität-GH Paderborn, D-33098 Paderborn, Germany

E-mail address: mat6jbs0ecci.upv.es

Departamento de ANálisis Matemático, Facultad de Matemáticas, Universidad de VAlencia, E-46100 BurJasot (VAlencia), Spain

E-mail address: fernand@rm.ci.uv.es 